\title{
PAH removal by simultaneous and sequential inoculation of Pseudomonas monteilii P26 and Gordonia sp. H19 in the presence of biostimulants
}

\author{
María Silvina Juárez Tomás ${ }^{\mathrm{a}, *}$, Marina Gabriela Carrasco ${ }^{\mathrm{a}, \mathrm{b}}$, Constanza Belén Lobo ${ }^{\mathrm{a}}$, \\ Mauricio Javier Alessandrello ${ }^{\mathrm{a}}$, Leandro Sánchez ${ }^{\mathrm{a}}$, Marcela Alejandra Ferrero ${ }^{\mathrm{a}, \mathrm{b}}$ \\ a Planta Piloto de Procesos Industriales Microbiológicos (PROIMI-CONICET), Av. Belgrano y Pje. Caseros, San Miguel de Tucumán, T4001MVB, Tucumán, Argentina \\ ${ }^{\mathrm{b}}$ Facultad de Bioquímica, Química y Farmacia, Universidad Nacional de Tucumán (UNT), Ayacucho 471, San Miguel de Tucumán, T4000INI, Tucumán, Argentina
}

\section{A R T I C L E I N F O}

\section{Keywords:}

Biostimulation

Hydrocarbon bioremoval

Bacterial mixed culture

Co-inoculation

Sequential inoculation

\begin{abstract}
A B S T R A C T
Biostimulation and bioaugmentation have been proposed as sustainable alternatives to physicochemical treatments for the environmental decontamination of polycyclic aromatic hydrocarbons (PAH). PAH-removing Pseudomonas monteilii P26 and Gordonia sp. H19, alone or combined with organic and inorganic biostimulants, were previously proposed as candidates to be included in biotechnological products to be used in eco-friendly decontamination processes. This work aims to study the effect of biostimulants on the growth, cell hydrophobicity and emulsifying activity of $P$. monteilii P26 and Gordonia sp. H19, and to evaluate the influence of selected biostimulants and bacterial inoculation procedure on PAH bioremoval. A complete factorial experimental design was applied to determine the effect of $\mathrm{KNO}_{3}, \mathrm{~K}_{2} \mathrm{HPO}_{4}$, corn steep liquor (CSL) and a PAH mixture on the growth, cell hydrophobicity and bioemulsifying activity of bacterial cultures. The kinetics of PAH bioremoval was evaluated in simultaneous and sequential cultures of P. monteilii P26 and Gordonia sp. H19 in the presence of selected biostimulants. CSL was the only nutrient that exerted positive effects on all responses evaluated, although these effects were not significant in all cases. In mixed cultures, maximum bioremoval of phenanthrene and pyrene was evidenced when the bacterial strains were simultaneously inoculated, independently of the presence or absence of CSL. These results support the biotechnological characterization of two PAH-removing autochthonous Patagonian strains.
\end{abstract}

\section{Introduction}

Environmental hazards lead to over a hundred human illnesses and traumas that cause around 12.6 million deaths every year worldwide (Prüss-Ustün et al., 2016). Environmental contamination by various substances such as metals, metalloids and organic compounds like hydrocarbons is a global ecological problem (Dror et al., 2016; PrüssUstün et al., 2016). Polycyclic aromatic hydrocarbons (PAH) are chemically stable, hydrophobic contaminants causing dramatic damages to living organisms (IARC, 2018). In the case of humans, exposition to $\mathrm{PAH}$ are related to increased risks of cancer development and adverse effects on embryos and fetuses (Prüss-Ustün et al., 2016; IARC, 2018).

Bioremediation is a sustainable alternative to eliminate contaminants from the environment or to convert them into innocuous or less toxic products. Bioremediation is a less expensive process than traditional physicochemical technologies (Lim et al., 2016; Zhao and
Liu, 2016). A biostimulation strategy consists in the addition of nutrients and other supplementary components to stimulate in situ growth or metabolism of organisms (Nikolopoulou et al., 2013; Hassanshahian et al., 2014). The bioaugmentation process is based on the use of exogenous organisms with proven decontamination abilities (Hassanshahian et al., 2014). Several studies have demonstrated that combined strategies of biostimulation and bioaugmentation could increase the efficacy of hydrocarbon bioremediation (Nikolopoulou et al., 2013; García-Delgado et al., 2015; Longang et al., 2016).

In the case of microorganisms with potential use in hydrocarbon bioremediation, microbial cell surface hydrophobicity and bioemulsifier production are interesting properties that could promote hydrocarbon removal (Andrade Silva et al., 2014; Ron and Rosenberg, 2014; Smułek et al., 2015). Through different mechanisms, these features favor the interaction of hydrophobic molecules with microbial cells (Nikolopoulou et al., 2013; Ron and Rosenberg, 2014). On the other

\footnotetext{
* Corresponding author. Planta Piloto de Procesos Industriales Microbiológicos (PROIMI)-CONICET, Avenida Belgrano y Pasaje Caseros, San Miguel de Tucumán, T4001MVB, Tucumán, Argentina.

E-mail addresses: sjuareztomas@proimi.org.ar (M.S. Juárez Tomás), maricarrasco377@gmail.com (M.G. Carrasco), blobo@proimi.org.ar (C.B. Lobo), mauricio.alessandrello@gmail.com (M.J. Alessandrello), 1sanchez@proimi.org.ar (L. Sánchez), ferreromar65@gmail.com (M.A. Ferrero).
} 
hand, different biostimulants (e.g., carbon, nitrogen and phosphorus sources) could increase cell hydrophobicity and bioemulsifying activity of several hydrocarbon-removing microorganisms (Zhao et al., 2011; Gudiña et al., 2015).

In previous studies, autochthonous bacterial strains were isolated from oil-polluted sediments from Patagonian coasts, Argentina, and characterized for their capability to remove different PAH under aerobic conditions (Isaac et al., 2013, 2015). Several Pseudomonas-actinobacteria co-cultures (e.g., Pseudomonas monteilii P26-Gordonia sp. H19) were proposed as potential candidates for their use in bioaugmentation processes (Isaac et al., 2015; Alessandrello et al., 2017a,b). In order to develop combined strategies of biostimulation and bioaugmentation for PAH bioremediation, the compatibility of selected PAHremoving bacteria with biostimulants should be determined. Biostimulant effects on microorganisms depend on species and strains; therefore, each strain must be specifically studied. In this work, the influence of different substances with potential use as biostimulants on the growth, cell surface hydrophobicity and bioemulsifying activity of P. monteilii P26 and Gordonia sp. H19 was studied. Simultaneous and sequential cultures of $P$. monteilii P26 and Gordonia sp. H19 were carried out to evaluate PAH bioremoval ability in the presence of the selected biostimulant.

\section{Materials and methods}

\subsection{Microorganisms and inoculum preparation}

P. monteilii P26 (HE798531) and Gordonia sp. H19 (LN680636) were maintained as frozen cultures (at $-20{ }^{\circ} \mathrm{C}$ ) in JPP broth added with $20 \%$ $\left(\mathrm{v} \mathrm{v}^{-1}\right)$ glycerol. JPP broth, which was previously designed in our laboratory for marine bacteria, contains a high salt concentration $(2 \% \mathrm{w}$ $\left.\mathrm{v}^{-1} \mathrm{NaCl}\right)$ and low amounts of nutrients $\left(\% \mathrm{w} \mathrm{v}^{-1}\right.$ : yeast extract, 0.1 ; meat peptone, 0.2) (Riva Mercadal et al., 2010).

Each stored bacterial strain was subcultured in JPP broth, at $30^{\circ} \mathrm{C}$ with agitation (180 rpm) for $24 \mathrm{~h}$ (P. monteilii P26) or $72 \mathrm{~h}$ (Gordonia sp. $\mathrm{H} 19)$. The inoculum of each culture was prepared in saline $(0.85 \% \mathrm{w}$ $\left.\mathrm{v}^{-1} \mathrm{NaCl}\right)$ adjusting the optical density at $600 \mathrm{~nm}\left(\mathrm{OD}_{600 \mathrm{~nm}}\right.$; Spectrophotometer SP-2000 UV, Spectrum, China; path length $10 \mathrm{~mm}$ ) to $0.9-1.1$.

\subsection{Effect of biostimulants on the growth, cell hydrophobicity and bioemulsifying activity of pure and mixed cultures of P. monteilii P26 and Gordonia sp. H19}

\subsubsection{Factorial experimental design}

In these experiments, an organic nitrogen and carbon source (corn steep liquor, CSL) and inorganic phosphorus and nitrogen sources $\left(\mathrm{K}_{2} \mathrm{HPO}_{4}\right.$ and $\mathrm{KNO}_{3}$ respectively) were assayed as potential biostimulants. A PAH mixture (phenanthrene and pyrene -Sigma Aldrich Co., St. Louis, MO, US- in acetone) was evaluated as a contaminant. A complete factorial design $2^{4}$ was applied to assess the influence of CSL ( 0 and $\left.10 \mathrm{~mL} \mathrm{~L}^{-1}\right), \mathrm{K}_{2} \mathrm{HPO}_{4}\left(0\right.$ and $\left.0.1 \mathrm{~g} \mathrm{~L}^{-1}\right), \mathrm{KNO}_{3}\left(0\right.$ and $\left.1 \mathrm{~g} \mathrm{~L}^{-1}\right)$ and $\mathrm{PAH}$ mixture ( 0 and $0.4 \mathrm{mM})$, on bacterial growth, cell hydrophobicity and bioemulsifying activity. Different combinations of biostimulants and PAH mixture (a total of 16 treatments) were assayed in JPP broth. The complete factorial design was carried out with each of three different sets of experiments (cultures): P. monteilii P26 pure culture, Gordonia sp. H19 pure culture and P. monteilii P26-Gordonia sp. H19 (P26-H19) coculture. The complete experimental design was performed three times on different days for each culture.

\subsubsection{Culture conditions and analytical determinations}

For pure and mixed cultures, $20 \mathrm{~mL}$ flasks containing $5 \mathrm{~mL}$ of each culture medium were used, and the inoculum of each strain was $5 \%(\mathrm{v}$ $\mathrm{v}^{-1}$ ). In culture media with $\mathrm{PAH}, \mathrm{PAH}$ mixture (phenanthrene and pyrene, $0.2 \mathrm{mM}$ each) in acetone was added before bacterial inoculation. For acetone evaporation, flasks were kept at room temperature for $15 \mathrm{~min}$. Flasks were incubated at $30^{\circ} \mathrm{C}$ with agitation $(180 \mathrm{rpm})$ for seven days. Then, bacterial growth, cell hydrophobicity and bioemulsifying activity were assayed. Instead of the conventional method of viable cell count, an indirect method $\left(\mathrm{OD}_{600 \mathrm{~nm}}\right.$ measurements) was used to determine bacterial growth. OD measurements constitute a rapid, inexpensive and reproducible technique, which is widely applied for microbial growth determination, mainly when multiple culture conditions are simultaneously assayed (Dalgaard et al., 1994; Juárez Tomás et al., 2002; Alessandrello et al., 2017a).

Cell surface hydrophobicity was determined by microbial adhesion to hydrocarbons (Rosenberg et al., 1980). This method, which allows the determination of the hydrophobic nature of the microbial surface, is based on the tendency of cells to migrate from an aqueous phase to a non-polar organic phase (the higher the hydrophobicity, the higher the affinity of cells for the non-polar organic solvent). Briefly, an aliquot of each culture was centrifuged $(8,000 \times g$ for $10 \mathrm{~min})$, washed and resuspended in saline to an $\mathrm{OD}_{600 \mathrm{~nm}}$ of $0.6 \pm 0.06$. N-hexadecane (Sigma Aldrich Co., St. Louis, MO, US) was added to cell suspensions; mixtures were vortexed for $1 \mathrm{~min}$ and allowed to stabilize for $15 \mathrm{~min}$. $\mathrm{OD}_{600 \mathrm{~nm}}$ of the aqueous phase was determined, and the percentage of hydrophobicity was calculated using the following expression:

Hydrophobicity $(\%)=\left[\left(\mathrm{OD}_{\text {initial }}-\mathrm{OD}_{\text {final }}\right) / \mathrm{OD}_{\text {initial }}\right] \times 100$

where $\mathrm{OD}_{\text {initial }}$ is the $\mathrm{OD}$ at the initial time of the hydrophobicity assay, and $\mathrm{OD}_{\text {final }}$ is the final $\mathrm{OD}$ at the end of the assay. Results were classified into three categories: low (0-35\%), medium (36-70\%) and high (71-100\%) hydrophobicity (Ocaña et al., 1999).

Bioemulsifying activity was evaluated by mechanical agitation of culture supernatants with kerosene, as previously described by Isaac et al. (2015). Briefly, an aliquot of each culture was centrifuged at $8,000 \times g$ for $10 \mathrm{~min}$. Cell-free supernatants were mixed with kerosene, vortexed for $2 \mathrm{~min}$ and allowed to stabilize for 1 and $24 \mathrm{~h}$. Negative and positive controls of emulsifying activity were each assayed culture medium (sterile broths) and Tween 20, respectively. The height of the emulsified layer ( $\mathrm{mm}$ ) and the total height of the liquid column (mm) were measured and the emulsification indexes at 1 and $24 \mathrm{~h}$ (EI-1 and EI-24, respectively) were calculated using the following expression:

EI $(\%)=\left(\mathrm{H}_{\mathrm{emulsion}} / \mathrm{H}_{\text {total }}\right) \times 100$

where $\mathrm{H}_{\mathrm{emulsion}}$ is the height of the emulsified layer, and $\mathrm{H}_{\text {total }}$ the total height of the liquid column. EI-24 results were classified into three categories: low (0-35\%), medium (36-70\%) and high (71-100\%) bioemulsifying activity.

Emulsion stability (ES) was calculated according to the following expression:

$\mathrm{ES}(\%)=(\mathrm{EI}-24 / \mathrm{EI}-1) \times 100$

According to Bosch et al. (1988), an emulsion is considered stable when its ES value is equal to or higher than $50 \%$.

2.3. Kinetics of $P A H$ removal in simultaneous and sequential cultures of $P$. monteilii P26 and Gordonia sp. H1, in the presence of the selected biostimulant

P. monteilii P26-Gordonia sp. H19 mixed cultures were carried out in JPP as the basal culture medium, with or without the selected biostimulant, and in the presence of the PAH mixture (phenanthrene and pyrene, $0.2 \mathrm{mM}$ each, in acetone). P. monteilii P26 and Gordonia sp. H19 inocula were prepared as described above. Although P. monteilii P26 and Gordonia sp. H19 are compatible strains (Isaac et al., 2015), they were inoculated ( $5 \% \mathrm{v} \mathrm{v}^{-1}$ each) either simultaneously ( $P$. monteilii P26 and Gordonia sp. H19 at initial time of culture) or sequentially ( $P$. monteilii P26 at initial time, and Gordonia sp. H19 after $72 \mathrm{~h}$ of culture) in $100 \mathrm{~mL}$ flasks containing $30 \mathrm{~mL}$ of the corresponding culture 
medium. The incubation of cultures was performed at $30^{\circ} \mathrm{C}$ with agitation $(180 \mathrm{rpm})$ for ten days.

At different times, entire reaction flasks were analyzed (three replicates). $30 \mathrm{~mL}$ acetone were added to each flask to homogenize the aqueous-organic mixture. Then, an aliquot was centrifuged $(6,300 \times g$ for $10 \mathrm{~min})$, and the supernatant was filtered $(0.22 \mu \mathrm{m}$ nylon filter) and stored at $-20^{\circ} \mathrm{C}$ until PAH quantification (Isaac et al., 2013, 2015; Alessandrello et al., 2017a). Control flasks without bacteria (three replicates) were used for each culture medium and sampling time in order to determine possible PAH abiotic loss.

PAH concentrations were quantified by reverse phase high-performance liquid chromatography (RP-HPLC), according to the methodology previously described by Isaac et al. (2013, 2015) and Alessandrello et al. (2017a). An Alliance e2695 (Waters Co., MA, USA) HPLC equipment coupled to a PDA detector (Waters 2998, Waters Corporation, MA, USA) was used. Filtered samples were automatically injected into a C18 Phenomenex Gemini HPLC column $(4.60 \times 250 \mathrm{~mm}$ $110 \AA$ pore size, $5 \mu \mathrm{m}$ particle size). The mobile phase was water:methanol (9:1) (solvent A) and methanol (solvent B). Each sample was evaluated under the following solvents program: initially, 20\% solvent $\mathrm{A}$ and $80 \%\left(\mathrm{v} \mathrm{v}^{-1}\right)$ solvent $\mathrm{B}$ for $10 \mathrm{~min}$, then $10 \%$ solvent $\mathrm{A}$ and $90 \%$ solvent B for $3 \mathrm{~min}$ and finally $100 \%$ solvent B for $7 \mathrm{~min}$. An external standard calibration curve was previously constructed by triplicate for each $\mathrm{PAH}$ and used for its quantification.

For each treatment and incubation time, a mass balance was applied to estimate the amount of each bioremoved PAH, as expressed in the following equation (Safdari et al., 2018):

$\mathrm{PAH}_{\mathrm{i}}=$ residual $\mathrm{PAH}_{\mathrm{tBC}}+$ removed $\mathrm{PAH}_{\mathrm{tAC}}+$ bioremoved $\mathrm{PAH}_{\mathrm{tBC}}$

where $\mathrm{PAH}_{\mathrm{i}}$ stands for $\mathrm{PAH}$ amount at the initial time of the removal assay, residual $\mathrm{PAH}_{\mathrm{tBC}}$ for $\mathrm{PAH}$ amount at time $\mathrm{t}$ of the simultaneous or sequential bacterial culture, removed $\mathrm{PAH}_{\mathrm{tAC}}$ for $\mathrm{PAH}$ amount that was removed by abiotic phenomena at time $t$ of incubation (i.e., it is the difference between $\mathrm{PAH}_{\mathrm{i}}$ and $\mathrm{PAH}$ amount in the abiotic control flask), and bioremoved $\mathrm{PAH}_{\mathrm{tBC}}$ for $\mathrm{PAH}$ amount that was removed by the bacterial culture at time $t$ of incubation. The percentage of each PAH amount was calculated according to the mass balance equation that was described above, considering $\mathrm{PAH}_{\mathrm{i}}$ equal to $100 \%$.

\subsection{Statistical analysis}

Results of $\mathrm{OD}_{600 \mathrm{~nm}}$, cell hydrophobicity percentages and EI-24, which were obtained in all the culture media of the experimental design $2^{4}$, were evaluated using a non-parametric analysis of variance (ANOVA) with the Kruskal-Wallis test. The statistical analysis was performed for each culture ( $P$. monteilii P26 and Gordonia sp. H19 pure cultures and P26-H19 mixed cultures). Pearson's correlation coefficients between hydrophobicity percentages, EI-24 and $\mathrm{OD}_{600} \mathrm{~nm}$ values were calculated. Bioremoval results were analyzed applying a general linear model of ANOVA to determine the main effects and interaction effects of the various factors (inoculation procedure, culture medium and incubation time) on each response assayed (phenanthrene or pyrene bioremoval). In this analysis, Tukey's test was applied to determine the significant differences between mean values, considering a p-value $<0.05$ as statistically significant. Analyses and plots were performed using InfoStat version 2016 and Minitab 17 Statistical Software.

\section{Results and discussion}

Nutrient supplementation has been proposed as a strategy to improve hydrocarbon degradation rate by indigenous and exogenous microorganisms involved in bioremediation processes (Czaplicki et al., 2018; Yang et al., 2018). Corn production is one of the leading agricultural activities in Argentina. CSL, a by-product of the corn wetmilling industry, is often discarded in spite of its various profitable uses. CSL is an inexpensive source of several nutrients (e.g., proteins, amino acids, vitamins, carbohydrates, organic acids, and minerals) used for the growth and metabolite production of microorganisms (Gudiña et al., 2015; Velázquez-Torres et al., 2015). We hypothesized that CSL and inorganic substances could be used to improve the growth and some interesting physiological properties of indigenous Patagonian $\mathrm{PAH}-$ removing bacteria and, consequently, to promote hydrocarbon removal.

\subsection{Effect of biostimulants on the growth, cell hydrophobicity and bioemulsifying activity of pure and mixed cultures of P. monteilii P26 and Gordonia sp. H19}

According to the results, biostimulants and PAH included in culture media differentially affected the growth, cell hydrophobicity and bioemulsifying activity of P. monteilii P26 and Gordonia sp. H19 when grown as pure as well as mixed cultures.

\subsubsection{Bacterial growth}

When comparing the growth of pure and mixed cultures in the presence of different biostimulants and PAH concentrations, $\mathrm{OD}_{600 \mathrm{~nm}}$ mean values of Gordonia sp. H19 were higher than those of $P$. monteilii P26 and P26-H19 co-cultures (Fig. 1). OD 600 nm values of P26-H19 cocultures were similar to those of $P$. monteilii P26 pure cultures. These results suggest that the $\mathrm{P} 26-\mathrm{H} 19$ co-cultures grow according to the growth of $P$. monteilii P26. In previous studies, the absence of growth inhibition by antagonistic substances between these bacterial strains was evidenced, suggesting that they can be associated in a co-culture (Isaac et al., 2015). It seems likely that other mechanisms such as nutrient competition negatively affected Gordonia sp. H19 growth in mixed cultures. Further studies need to be carried out to evaluate the interaction mechanisms between the microorganisms involved in the P26-H19 mixed culture.

Among the different factors assayed, only CSL and PAH significantly affected bacterial growth. When comparing bacterial growth in the sixteen culture media assayed, the increase in CSL concentration had a positive effect on the $\mathrm{OD}_{600} \mathrm{~nm}$ values of $P$. monteilii P26 pure cultures and P26-H19 co-cultures (Fig. 1). Similarly, the growth of Pseudomonas sp. LP1 and Pseudomonas aeruginosa LP5 (two pyrene-degrading strains) was promoted by the addition of CSL in the culture medium (Obayori et al., 2010). In a microcosm assay, bacterial biomass significantly increased when CSL was added to oil-polluted soils (El-Gendy and Farah, 2011). However, the presence of $\mathrm{N}$ and $\mathrm{P}$ inorganic sources $\left(\mathrm{KNO}_{3}\right.$ and $\mathrm{KH}_{2} \mathrm{PO}_{4}$ ) did not significantly favor the growth of either P. monteilii P26 or Gordonia sp. H19 in pure or mixed cultures. In a similar way, a microbial consortium from seawater showed lower numbers of hydrocarbon-degrading cells and growth rates in the presence of only $\mathrm{N}$ and $\mathrm{P}$ inorganic sources than in the presence of organic compounds (e.g., uric acid, lecithin and rhamnolipid biosurfactants) as biostimulants (Nikolopoulou et al., 2013).

The presence of PAH in culture media significantly favored the growth of Gordonia sp. H19; however, it exerted an adverse effect on the growth of $P$. monteilii P26 and P26-H19 co-cultures. The ability to remove phenanthrene and pyrene by planktonic $P$. monteilii P26 and Gordonia sp. H19 cells (in pure cultures), respectively, was previously demonstrated (Isaac et al., 2013, 2015). Moreover, phenanthrene and pyrene stimulated the growth of biofilm-immobilized P. monteilii P26 (Isaac et al., 2017). However, the results obtained in this work suggest that, under the experimental conditions assayed, the possible PAH bioremoval did not generate carbon and energy for P. monteilii P26 growth. This relation between contaminant and microbial growth is a type of co-metabolic process (Johnsen et al., 2005). In the case of $P$. monteilii P26, the results of co-metabolism can be due to the presence of carbon sources in the JPP broth (e.g., amino acids from yeast extract 

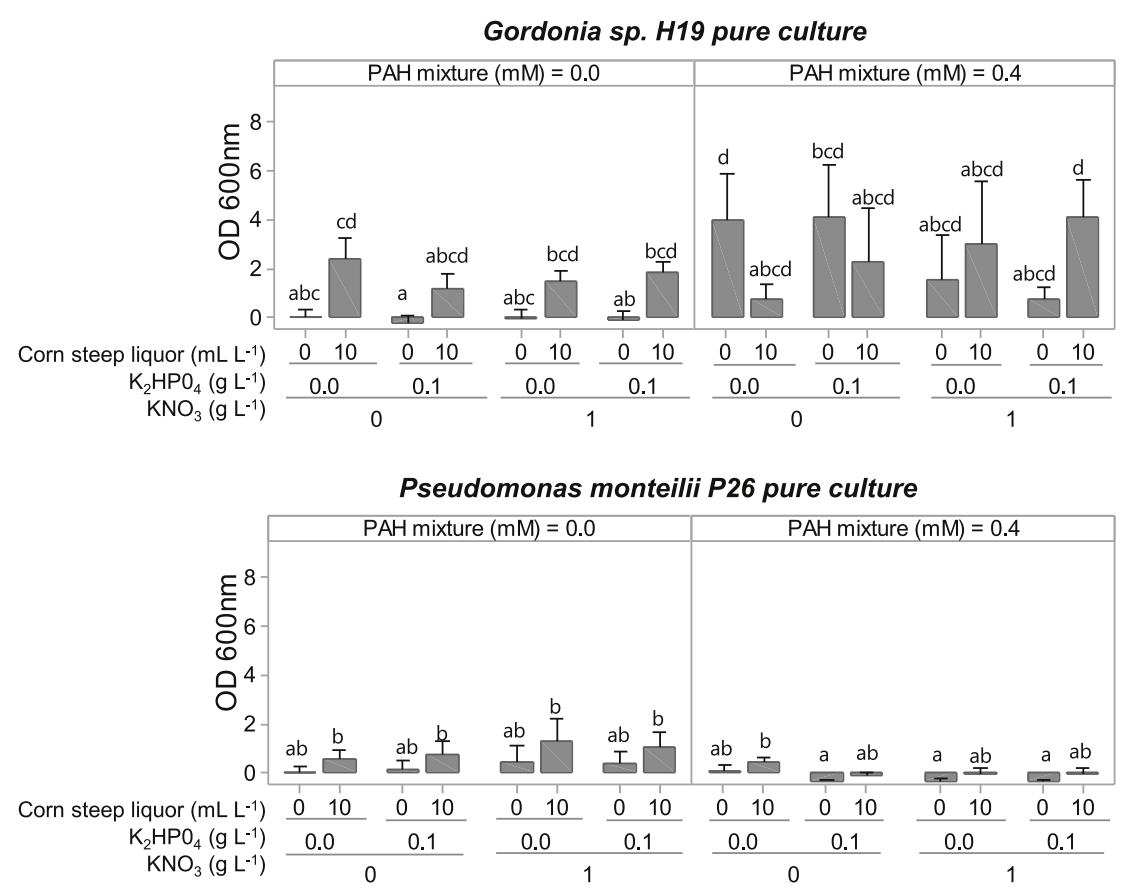

Pseudomonas monteilii P26-Gordonia sp. H19 mixed culture

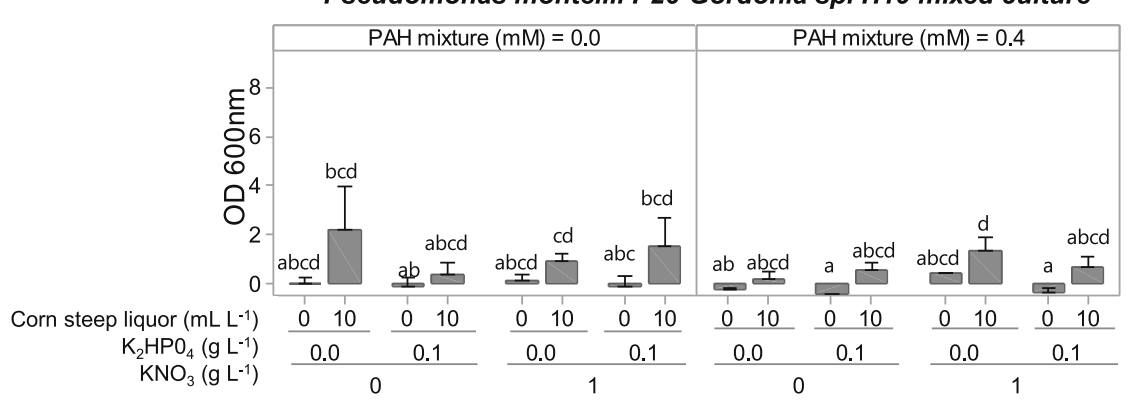

Fig. 1. Growth of Pseudomonas monteilii P26 and Gordonia sp. $\mathrm{H} 19$ in pure and mixed cultures at different concentrations of biostimulants $\left(\mathrm{KNO}_{3}\right.$, $\mathrm{K}_{2} \mathrm{HPO}_{4}$ and corn steep liquor) and a polycyclic aromatic hydrocarbon (PAH) mixture (phenanthrene and pyrene) added to JPP broth (basal medium), after seven days of incubation at $30^{\circ} \mathrm{C}$ with agitation. Data are expressed as the mean values of $\mathrm{OD}_{600 \mathrm{~nm}}+$ standard error. For each culture (Gordonia sp. H19 and P. monteilii P26 pure cultures and P. monteilii P26- Gordonia sp. H19 mixed cultures), the growth in JPP broth without PAH or biostimulants was considered as base line. Therefore, this base line was subtracted to all the $\mathrm{OD}_{600} \mathrm{~nm}$ values. Different letters indicate statistically significant differences $(\mathrm{p}<0.05)$ in $\mathrm{OD}_{600 \mathrm{~nm}}$ values between different treatments (culture media) for each bacterial culture according to the KruskalWallis test. and meat peptone). On the contrary, Gordonia sp. H19 seems to remove $\mathrm{PAH}$ by a metabolic process, because its growth was higher in the presence of phenanthrene and pyrene, suggesting that these substrates can be used as carbon and energy source for bacterial biomass production (Becker and Seagren, 2010).

In a microcosm assay, biofilm-immobilized mixed cultures of $P$. monteilii P26 and Gordonia sp. H19 were able to efficiently remove crude oil from artificial sea water (a medium containing only mineral salts) (Alessandrello et al., 2017b). The bioremoval results of $P$. monteilii P26 and Gordonia sp. H19 in the presence of only essential minerals and crude oil (source of carbon) suggested that these bacteria are effective for hydrocarbon degradation and appropriate to be used for bioremediation (Alessandrello et al., 2017b). However, the PAH bioremoval rate can increase in culture media supplemented with nutrients, including additional carbon sources (Teng et al., 2010). In this work, JPP broth was used as basal medium on the basis of the results obtained in previous studies in which PAH aerobic bioremoval efficiency in JPP was higher than that in a minimum mineral medium (Isaac, 2014).

\subsubsection{Cell surface hydrophobicity}

The results obtained indicated that cell hydrophobicity percentages of Gordonia sp. H19 were higher than those of P. monteilii P26 and P26-H19 co-cultures (Fig. 2). In most culture media, Gordonia sp. H19 presented either medium or high cell hydrophobicity (mean values 50-81\%). In contrast, $P$. monteilii P26 and P26-H19 co-cultures showed low cell hydrophobicity in all culture media (mean values $0-15 \%$ ).
Similarly, Gordonia sp. JC11, a marine lubricant-degrading strain showed higher cell hydrophobicity than Pseudomonas strains (Chanthamalee and Luepromchai, 2012).

On the other hand, hydrophobicity values of Gordonia sp. H19 and $P$. monteilii P26 pure cultures were significantly higher in some culture media containing CSL and PAH simultaneously compared to other media with no PAH or CSL. In P26-H19 co-cultures, significantly higher hydrophobicity percentages were observed in several culture media containing PAH, especially when compared with media without hydrocarbons. These results suggest that, mainly in the presence of PAH, these bacteria could modify some of their surface characteristics (e.g., through a change in the chemical structure of their cell surface) in order to adapt themselves to hydrophobic substances (Schmutzler et al., 2016). A higher cell hydrophobicity could promote a close contact between bacterial cells and hydrocarbons (Hesham et al., 2014).

\subsubsection{Bioemulsifying activity}

The ability to form and stabilize emulsions is a microbial strategy to increase PAH solubility and facilitate their biodegradation (Hesham et al., 2014; Gudiña et al., 2015; Tian et al., 2016). The bioemulsifying activity of Gordonia sp. H19 culture supernatants (EI-24 mean values between 3 and 23\%) was lower than that of P. monteilii P26 and P26-H19 co-cultures (EI-24 mean values around 10-40\%) (Fig. 3). EI24 values of Gordonia sp. H19 in two culture media with PAH (JPP with $10 \mathrm{mLL}^{-1} \mathrm{CSL}$ and $0.1 \mathrm{gL}^{-1} \mathrm{~K}_{2} \mathrm{HPO}_{4}$, and JPP with $1 \mathrm{gL}^{-1} \mathrm{KNO}_{3}$ ) were significantly higher than the value found in a culture medium 

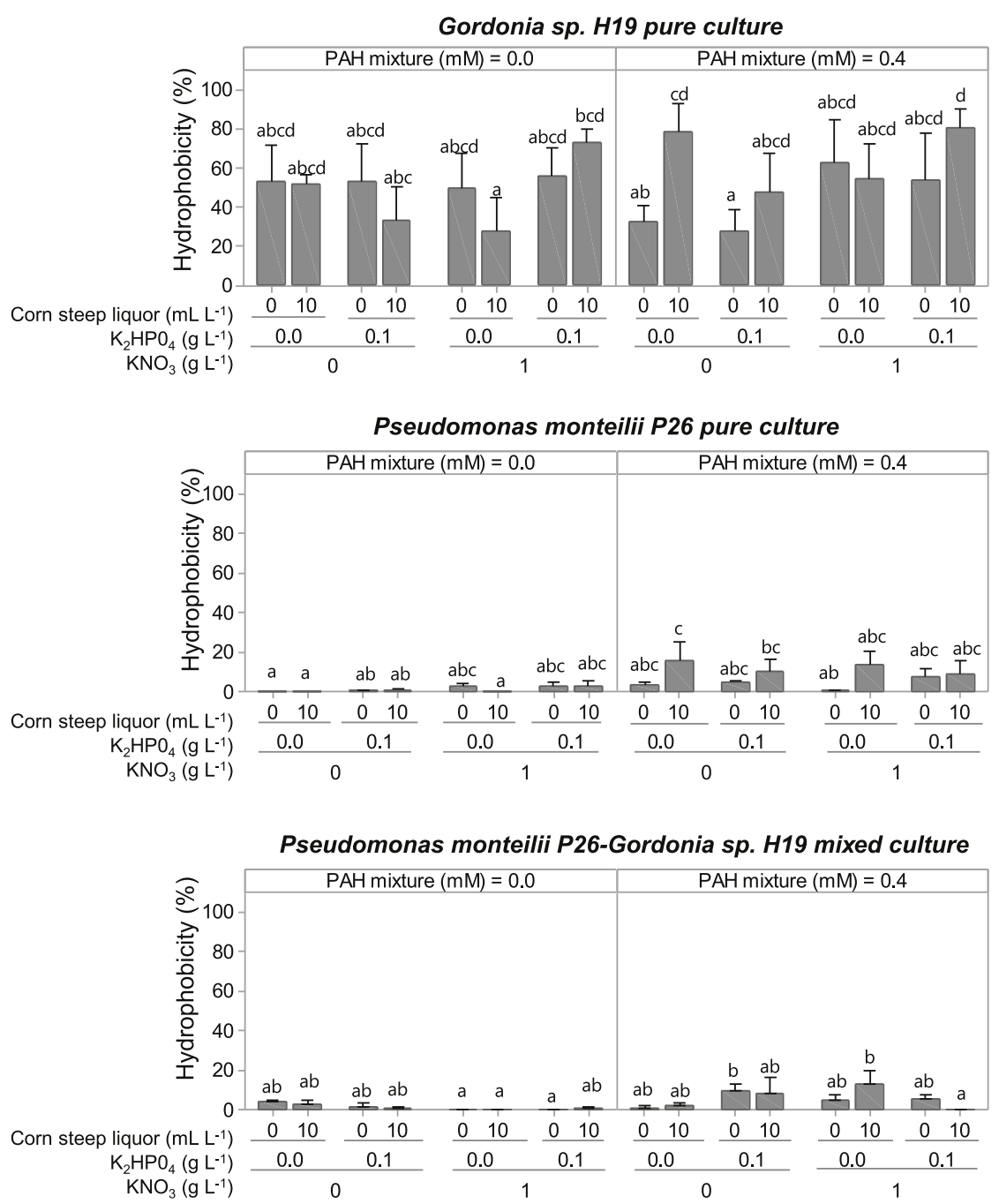

Fig. 2. Cell hydrophobicity of Pseudomonas monteilii P26 and Gordonia sp. H19 in pure and mixed cultures at different concentrations of biostimulants $\left(\mathrm{KNO}_{3}, \mathrm{~K}_{2} \mathrm{HPO}_{4}\right.$ and corn steep liquor) and a polycyclic aromatic hydrocarbon (PAH) mixture (phenanthrene and pyrene) added to JPP broth (basal medium), after seven days of incubation at $30{ }^{\circ} \mathrm{C}$ with agitation. Data are expressed as the mean values of cell hydrophobicity percentages + standard error. Different letters indicate statistically significant differences $(\mathrm{p}<0.05)$ in the hydrophobicity percentages between the different treatments (culture media) for each bacterial culture, according to the Kruskal-Wallis test. without PAH (JPP with $1 \mathrm{~g} \mathrm{~L}^{-1} \mathrm{KNO}_{3}$ ).

A significant positive effect of the presence of CSL on EI-24 values was evidenced only in P. monteilii P26. Similarly, Gudiña et al. (2015) demonstrated the positive effects of CSL on bacterial bioemulsifying activity of $P$. aeruginosa \#112, an autochthonous strain from an oil field. High IE-24 values (about 60\%) were evidenced when sugarcane molasses (an inexpensive by-product) was combined with CSL, without the supplementation with inorganic salts or expensive complex organic nutrients (Gudiña et al., 2015). In P26-H19 co-cultures, significantly higher EI-24 values in culture media with $\mathrm{K}_{2} \mathrm{HPO}_{4}$ and without $\mathrm{KNO}_{3}$ were observed, independently of the presence of CSL and/or PAH. In all the culture media assayed, emulsions formed with supernatants of Gordonia sp. H19 and P. monteilii P26 pure and mixed cultures were stable, with ES values higher than 50\% (data not shown).

\subsubsection{Relationships between hydrophobicity, bioemulsifying properties and growth}

When analyzing cell hydrophobicity and bioemulsifying activity data from all the cultures assayed (Gordonia sp. H19 and P. monteilii P26, and P26-H19 co-cultures), a significant negative linear correlation between the two properties was evidenced (Pearson's correlation value $=-0.422, \mathrm{p}<0.05$ ) (Fig. S1). On the whole, $P$. monteilii P26 and P26-H19 co-cultures showed low hydrophobicity and moderate bioemulsifying activity, while Gordonia sp. H19 exhibited higher hydrophobicity and poor bioemulsifying activity (Figs. 2 and 3). The results obtained indicate that P. monteilii P26 and Gordonia sp. H19 differ in the properties that allow them to interact with hydrophobic substrates. These results suggest that the mechanisms through which the bacterial strains assayed establish contact with PAH for subsequent bioremoval are different. PAH-cell contact is necessary to start the aerobic bacterial degradation of $\mathrm{PAH}$, which is a biochemical process involving cell surface-associated oxygenases (Ron and Rosenberg, 2002).

However, when evaluating the relationships between cell hydrophobicity and bioemulsifying activity for each strain and the co-culture in the different culture media, negative but no significant correlations were obtained (Pearson's correlation value $=-0.256, \mathrm{p}=0.158$ for Gordonia sp. H19; Pearson's correlation value $=-0.12, \mathrm{p}=0.514$ for P. monteilii P26, and Pearson's correlation value $=-0.215, \mathrm{p}=0.237$ for P26-H19 co-cultures).

Correlation analyses for all the cultures assayed including only results obtained in culture media with PAH indicated that the relationship between growth and bioemulsifying activity was non-significantly negative (Pearson's correlation value $=-0.246, p=0.091$ ), while the relationship between growth and cell hydrophobicity was significantly positive (Pearson's correlation value $=0.425 ; \mathrm{p}=0.003$ ). Similarly, the growth of Sphingomonas koreensis strain ASU-06 -in a mineral salt medium with several PAH- was higher at higher cell surface hydrophobicity, suggesting that this surface property could favor PAH use as a carbon source (Hesham et al., 2014). 


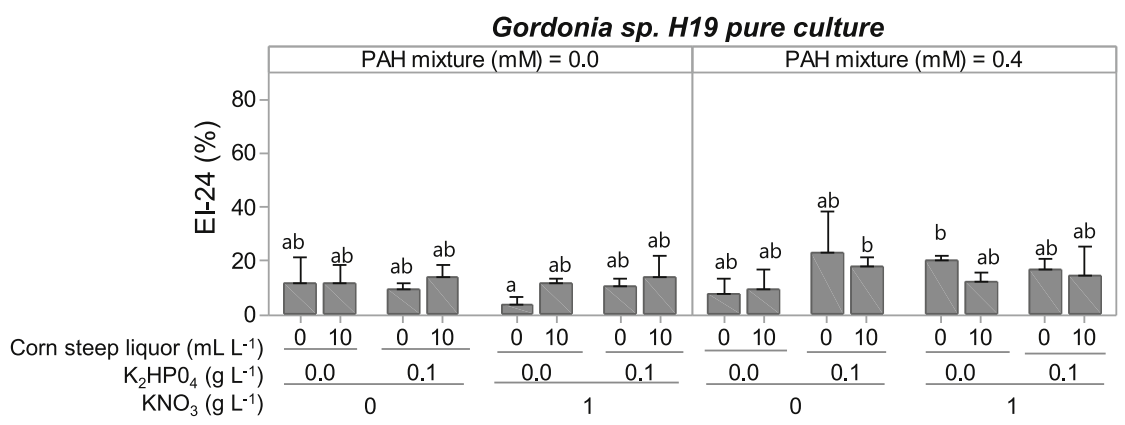

Pseudomonas monteilii P26 pure culture
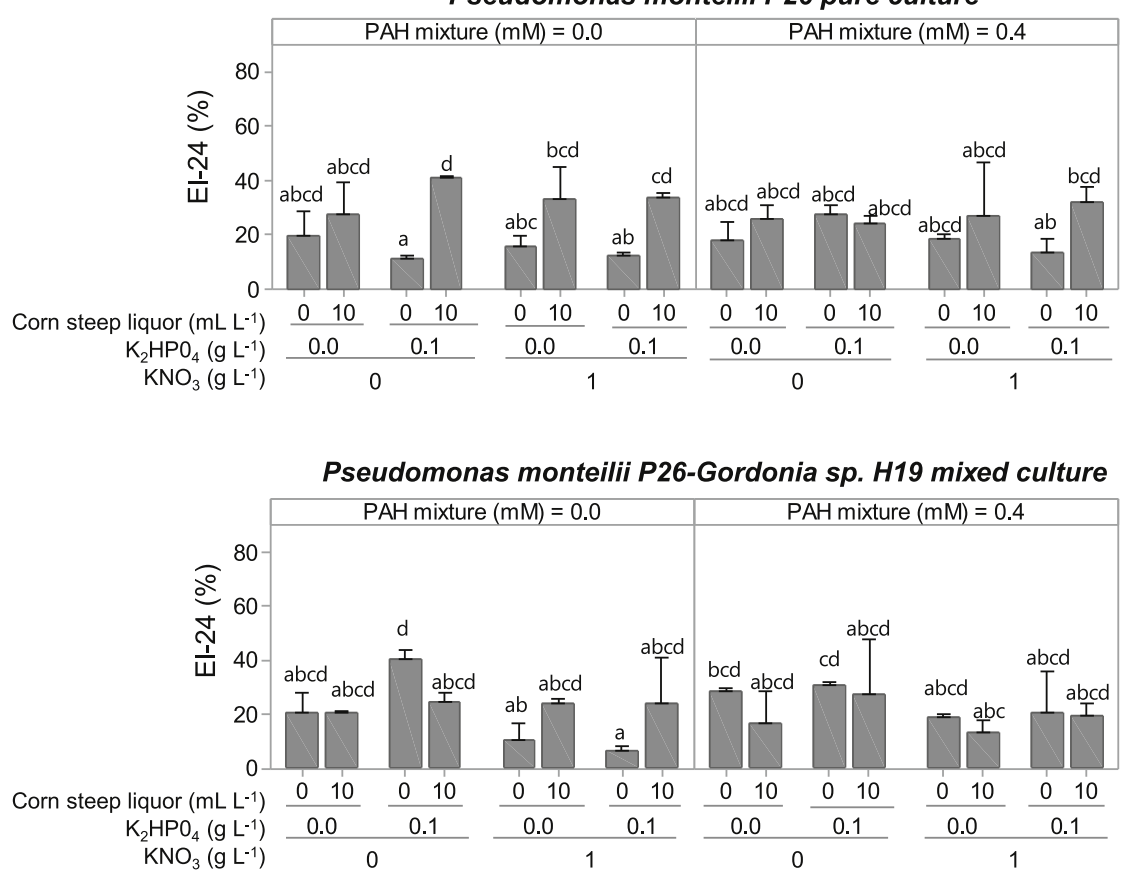

Fig. 3. Bioemulsifying activity of Pseudomonas monteilii P26 and Gordonia sp. H19 in pure and mixed cultures at different concentrations of biostimulants $\left(\mathrm{KNO}_{3}, \mathrm{~K}_{2} \mathrm{HPO}_{4}\right.$ and corn steep liquor) and a polycyclic aromatic hydrocarbon $(\mathrm{PAH})$ mixture (phenanthrene and pyrene) added to JPP broth (basal medium), after seven days of incubation at $30{ }^{\circ} \mathrm{C}$ with agitation. Data are expressed as the mean values of emulsification indexes at $24 \mathrm{~h}$ (EI-24, $\%)+$ standard error. Different letters indicate statistically significant differences $(\mathrm{p}<0.05)$ in the emulsification indexes at $24 \mathrm{~h}$ between the different treatments (culture media) for each bacterial culture according to the Kruskal-Wallis test.
3.2. Determination of the kinetics of PAH removal in simultaneous and sequential cultures of P. monteilii P26 and Gordonia sp. H19 in the presence of the selected biostimulant

The kinetics of PAH removal of P26-H19 cultures in JPP broth with or without CSL was evaluated on the basis of the significant effects, especially of CSL and PAH, evidenced on the various P. monteilii P26 and Gordonia sp. H19 properties in either pure or mixed cultures. Isaac et al. (2015) reported that Pseudomonas-actinobacteria mixed cultures removed a PAH complex mixture more efficiently than axenic cultures. In the co-cultures, maximum decrease in pyrene concentration, which was mainly caused by actinobacteria catabolism, was simultaneous to the maximum bioemulsifier production by Pseudomonas strains (Isaac et al., 2015).

In this work, simultaneous and sequential inoculations of $P$. monteilii P26 and Gordonia sp. H19 to prepare the mixed cultures were performed. In the sequential inoculation, the aim was first to allow bioemulsifier production -which could increase PAH solubility and bioavailability- and phenanthrene removal by $P$. monteilii P26. Then, bioemulsifier and metabolites produced by $P$. monteilii P26 could favor pyrene removal by Gordonia sp. H19. The proposed strategy is another type of co-metabolic process, in which initial co-metabolic transformations in axenic cultures can promote subsequent transformations by other microorganisms in mixed cultures (Becker and Seagren, 2010).

When analyzing the results of phenanthrene bioremoval, significant differences were evidenced at different incubation times and inoculation procedures, but not between culture media (JPP with or without CSL) (Fig. 4a). In simultaneous cultures in JPP, significantly higher phenanthrene bioremoval percentages (mean value, 49\%) were observed after seven and ten days of incubation compared to the initial time. However, in all other culture conditions assayed, a significantly higher bioremoval of phenanthrene was determined only after ten days of incubation. In JPP medium, phenanthrene bioremoval percentages after three days of sequential cultures were significantly lower than those after seven and ten days of simultaneous cultures.

Pyrene bioremoval was significantly affected by the incubation time and inoculation procedure of the bacteria included in the mixed culture (Fig. 4b). Moreover, the interaction effect among the three factors assayed was significant, indicating that the variation in pyrene bioremoval percentages at different incubation times was dependent both on the culture medium and on the inoculation system. Sequential inoculation of $P$. monteilii P26 and Gordonia sp. H19 in JPP medium negatively affected the amount of removed pyrene at all incubation times assayed. When comparing the results obtained in sequential cultures in different culture media, pyrene bioremoval percentages were slightly (at days 3 and 10) or significantly (at day 7) higher in JPP broth with CSL than without CSL. It seems likely that the presence of CSL in the culture medium promoted pyrene catabolism by the two bacterial strains of the culture despite the initial absence of Gordonia sp. H19. Further complementary studies will be performed to verify the 
a

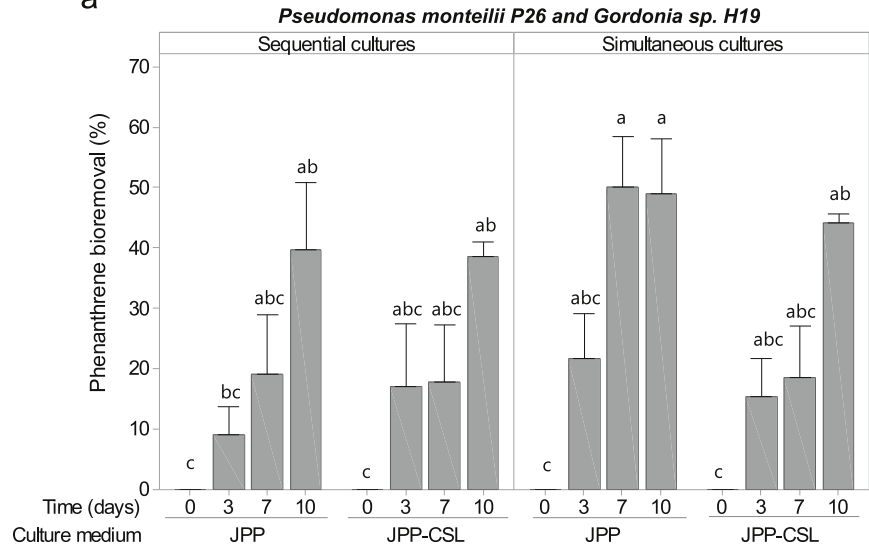

b

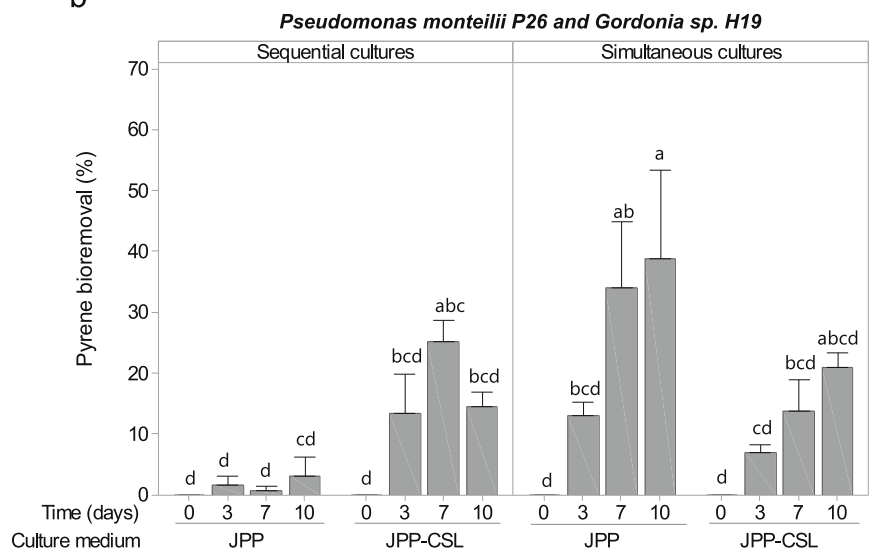

Fig. 4. Kinetics of phenanthrene (a) and pyrene (b) bioremoval by simultaneous and sequential cultures of Pseudomonas monteilii P26 and Gordonia sp. H19 in JPP broth and JPP supplemented with $10 \mathrm{~mL} \mathrm{~L}^{-1}$ corn steep liquor (JPP-CSL) at $30{ }^{\circ} \mathrm{C}$ with agitation. Data are expressed as the mean values of phenanthrene or pyrene bioremoval percentages + standard error. Different letters indicate statistically significant differences $(p<0.05)$ in bioremoval percentages between different inoculation procedures, culture media and incubation times for each hydrocarbon, according to Tukey's test.

hypotheses proposed.

When P. monteilii P26 and Gordonia sp. H19 were simultaneously inoculated in JPP culture medium, significantly higher pyrene bioremoval percentages (mean value, 39\%) were evidenced after seven and ten days of incubation, compared to the values determined at all the incubation times of sequential cultures in JPP medium. These results suggest that, under these culture conditions, the simultaneous presence of the two bacteria at initial time of $\mathrm{P} 26-\mathrm{H} 19$ mixed cultures is required to promote their optimal metabolic cooperation, which allows improving pyrene bioremoval efficiency. In contrast, in JPP medium with CSL, no significant differences in pyrene bioremoval were observed between sequential and simultaneous cultures.

Fig. 5 shows the results of mass balance assessment at the end of the removal assay. The mass balance equation described above (item 2.3.) was used to calculate the percentage of residual PAH, bioremoved PAH and PAH that was removed by abiotic processes in each culture condition assayed. The amount of phenanthrene that was removed by abiotic processes (from 4 to $12 \%$ ) was higher than that of pyrene (from 0 to $8 \%$ ). Moreover, phenanthrene and pyrene abiotic losses were higher in JPP-CSL than in JPP. Probably, some components present in CSL bind some hydrocarbons and slightly decrease their bioavailability for bacterial metabolism.

When analyzing the PAH amounts that were removed by bacterial a

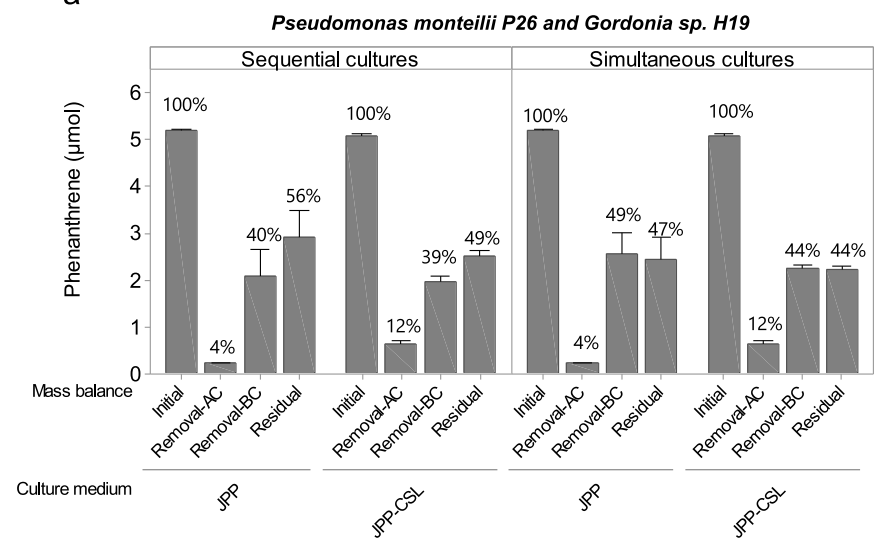

b

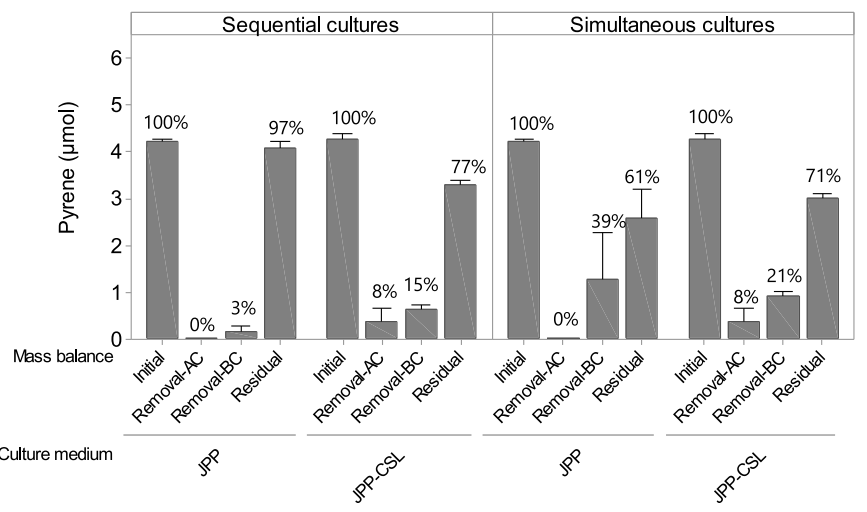

Fig. 5. Mass balance of phenanthrene (a) and pyrene (b) at the end of the removal assay (10 days) by simultaneous and sequential cultures of Pseudomonas monteilii P26 and Gordonia sp. H19 in JPP broth and JPP supplemented with $10 \mathrm{~mL} \mathrm{~L}^{-1}$ corn steep liquor (JPP-CSL). Initial: phenanthrene or pyrene amount in reaction flasks at the initial time of the removal assay. Removal-AC: phenanthrene or pyrene amount that was removed by abiotic phenomena. Removal-BC: phenanthrene or pyrene amount that was removed by the bacterial culture. Residual: remaining phenanthrene or pyrene amount in reaction flasks of bacterial culture. Data are expressed as the mean values of phenanthrene or pyrene $\mu \mathrm{mol}+$ standard error. The percentage of each hydrocarbon amount respecting to initial hydrocarbon amount in reaction flasks is indicated on each bar.

cultures at the end of the removal assay, the amounts of bioremoved phenanthrene were similar in the different culture media and inoculation procedures. However, the amounts of bioremoved pyrene in simultaneous cultures were higher than in sequential cultures. The lowest percentage of bioremoved pyrene was determined in sequential cultures in JPP without CSL. On the other hand, the amounts of bioremoved phenanthrene were higher than those of pyrene in all the culture conditions assayed. The lowest differences between amounts of bioremoved phenanthrene and pyrene was observed in simultaneous cultures in JPP broth.

\section{Conclusions}

This study contributes to the knowledge of the biotechnological properties and compatibility with biostimulants of hydrocarbon-removing bacteria for their potential application to environmental bioremediation. Further studies should be performed to select biostimulants that effectively promote PAH bioremoval by beneficial bacteria, with a view to the development of formulations for their use in complementary bioaugmentation and biostimulation strategies. 


\section{Declaration of interest}

The authors have no conflict of interests regarding the publication of this article.

\section{Acknowledgements}

This work was supported by grants of Consejo Nacional de Investigaciones Científicas y Técnicas, CONICET, Argentina [PIP 20130470 and P-UE 2016-0012] and Agencia Nacional de Promoción Científica y Tecnológica, ANPCyT [PICT 2015-1867].

\section{Appendix A. Supplementary data}

Supplementary data to this article can be found online at https:// doi.org/10.1016/j.ibiod.2019.104752.

\section{References}

Alessandrello, M.J., Juárez Tomás, M.S., Isaac, P., Vullo, D.L., Ferrero, M.A., 2017a. PAH removal by immobilized bacterial cells-support systems using low-cost culture media for biomass production. Int. Biodeterior. Biodegrad. 120, 6-14. https://doi.org/10. 1016/j.ibiod.2017.01.038.

Alessandrello, M.J., Juárez Tomás, M.S., Raimondo, E.E., Vullo, D.L., Ferrero, M.A., 2017b. Petroleum oil removal by immobilized bacterial cells on polyurethane foam under different temperature conditions. Mar. Pollut. Bull. 122, 156-160. https://doi. org/10.1016/j.marpolbul.2017.06.040.

Andrade Silva, N.R., Luna, M.A., Santiago, A.L., Franco, L.O., Silva, G.K., de Souza, P.M., Okada, K., Albuquerque, C.D., Alves da Silva, C.A., Campos-Takaki, G.M., 2014. Biosurfactant-and-bioemulsifier produced by a promising Cunninghamella echinulata isolated from Caatinga soil in the northeast of Brazil. Int. J. Mol. Sci. 15, 15377-15395. https://doi.org/10.3390/ijms150915377.

Becker, J.G., Seagren, E.A., 2010. Bioremediation of hazardous organics. In: Mitchell, R., Gu, J.-D. (Eds.), Environmental Microbiology, second ed. Wiley-Blackwell, New Jersey, pp. 177-212.

Bosch, M.P., Robert, M., Mercade, M.E., Espuny, M.J., Parra, J.L., Guinea, J., 1988. Surface-active compounds on microbial cultures. Tenside Surfactants Deterg. 25, 208-211.

Czaplicki, L.M., Dharia, M., Cooper, E.M., Ferguson, P.L., Gunsch, C.K., 2018. Evaluating the mycostimulation potential of select carbon amendments for the degradation of a model PAH by an ascomycete strain enriched from a superfund site. Biodegradation 29, 463-471. https://doi.org/10.1007/s10532-018-9843-z.

Chanthamalee, J., Luepromchai, E., 2012. Isolation and application of Gordonia sp. JC11 for removal of boat lubricants. J. Gen. Appl. Microbiol. 58, 19-31. https://doi.org/ 10.2323/jgam.58.19.

Dalgaard, P., Ross, T., Kamperman, L., Neumeyer, K., McMeekin, T.A., 1994. Estimation of bacterial growth rates from turbidimetric and viable count data. Int. J. Food Microbiol. 23, 391-404. https://doi.org/10.1016/0168-1605(94)90165-1.

Dror, I., Yaron, B., Berkowitz, B., 2016. Microchemical contaminants as forming agents of anthropogenic soils. Ambio 46, 109-120. https://doi.org/10.1007/s13280-0160804-7.

El-Gendy, N.S., Farah, J.Y., 2011. Kinetic modeling and error analysis for decontamination of different petroleum hydrocarbon components in biostimulation of oily soil microcosm. Soil Sediment Contam. 20, 432-446. https://doi.org/10.1080/ 15320383.2011.571525.

García-Delgado, C., D'Annibale, A., Pesciaroli, L., Yunta, F., Crognale, S., Petruccioli, M., Eymar, E., 2015. Implications of polluted soil biostimulation and bioaugmentation with spent mushroom substrate (Agaricus bisporus) on the microbial community and polycyclic aromatic hydrocarbons biodegradation. Sci. Total Environ. 508, 20-28. https://doi.org/10.1016/j.scitotenv.2014.11.046.

Gudiña, E.J., Rodrigues, A.I., Alves, E., Domingues, M.R., Teixeira, J.A., Rodrigues, L.R., 2015. Bioconversion of agro-industrial by-products in rhamnolipids toward applications in enhanced oil recovery and bioremediation. Bioresour. Technol. 177, 87-93. https://doi.org/10.1016/j.biortech.2014.11.069.

Hassanshahian, M., Emtiazi, G., Caruso, G., Cappello, S., 2014. Bioremediation (bioaugmentation/biostimulation) trials of oil polluted seawater: a mesocosm simulation study. Mar. Environ. Res. 95, 28-38. https://doi.org/10.1016/j.marenvres.2013.12. 010 .

Hesham, A.El-L., Mawad, A.M., Mostafa, Y.M., Shoreit, A., 2014. Biodegradation ability and catabolic genes of petroleum-degrading Sphingomonas koreensis strain ASU-06 isolated from Egyptian oily soil. BioMed Res. Int 2014, e127674. https://doi.org/10. $1155 / 2014 / 127674$

International Agency for Research on Cancer (IARC), 2018. Monographs on the evaluation of carcinogenic risks to humans. Agents Classified by the IARC Monographs. http://monographs.iarc.fr/ENG/Classification/List_of_Classifications.pdf accessed 20 December 2018.

Isaac, P., Alessandrello, M.J., Macedo, A.J., Estévez, M.C., Ferrero, M.A., 2017. Pre- exposition to polycyclic aromatic hydrocarbons (PAHs) enhance biofilm formation and hydrocarbon removal by native multi-species consortium. J. Environ. Chem. Eng 5, 1372-1378. https://doi.org/10.1016/j.jece.2017.02.031.

Isaac, P., 2014. Caracterización fisiológica y genética de bacterias marinas hidrocarbonoclásticas para su utilización en biotecnologías aplicadas a la restauración de sitios contaminados con hidrocarburos. PhD thesis. Universidad Nacional de Tucumán, Tucumán, Argentina.

Isaac, P., Martínez, F.L., Bourguignon, N., Sánchez, L.A., Ferrero, M.A., 2015. Improved PAHs removal performance by a defined bacterial consortium of indigenous Pseudomonas and actinobacteria from Patagonia, Argentina. Int. Biodeterior. Biodegrad. 101, 23-31. https://doi.org/10.1016/j.ibiod.2015.03.014.

Isaac, P., Sánchez, L.A., Bourguignon, N., Cabral, M.E., Ferrero, M.A., 2013. Indigenous PAH-degrading bacteria from oil-polluted sediments in Caleta Cordova, Patagonia Argentina. Int. Biodeterior. Biodegrad. 82, 207-214. https://doi.org/10.1016/j. ibiod.2013.03.009.

Johnsen, A.R., Wick, L.Y., Harms, H., 2005. Principles of microbial PAH-degradation in soil. Environ. Pollut. 133, 71-84. https://doi.org/10.1016/j.envpol.2004.04.015.

Juárez Tomás, M.S., Bru, E., Wiese, B., De Ruiz Holgado, A.P., Nader-Macías, M.E., 2002. Influence of $\mathrm{pH}$, temperature and culture media on the growth and bacteriocin production of vaginal Lactobacillus salivarius CRL 1328. J. Appl. Microbiol. 93, 714-724. https://doi.org/10.1046/j.1365-2672.2002.01753.x.

Lim, M.W., Lau, E.V., Poh, P.E., 2016. A comprehensive guide of remediation technologies for oil contaminated soil - present works and future directions. Mar. Pollut. Bull. 109, 14-45. https://doi.org/10.1016/j.marpolbul.2016.04.023.

Longang, A., Buck, C., Kirkwood, K.M., 2016. Halotolerance and effect of salt on hydrophobicity in hydrocarbon-degrading bacteria. Environ. Technol. 37, 1133-1140. https://doi.org/10.1080/09593330.2015.1102333.

Nikolopoulou, M., Pasadakis, N., Kalogerakis, N., 2013. Evaluation of autochthonous bioaugmentation and biostimulation during microcosm-simulated oil spills. Mar. Pollut. Bull. 72, 165-173. https://doi.org/10.1016/j.marpolbul.2013.04.007.

Obayori, O.S., Adebusoye, S.A., Ilori, M.O., Oyetibo, G.O., Omotayo, A.E., Amund, O.O., 2010. Effects of corn steep liquor on growth rate and pyrene degradation by Pseudomonas strains. Curr. Microbiol. 60, 407-411. https://doi.org/10.1007/s00284009-9557-X.

Ocaña, V.S., Bru, E., de Ruiz Holgado, A.P., Nader-Macías, M.E., 1999. Surface characteristics of lactobacilli isolated from human vagina. J. Gen. Appl. Microbiol. 45, 203-212. https://doi.org/10.2323/jgam.45.203.

Prüss-Ustün, A., Wolf, J., Corvalán, C., Bos, R., Neira, M., 2016. Preventing Disease through Healthy Environments: a Global Assessment of the Burden of Disease from Environmental Risks. World Health Organization, Switzerland.

Riva Mercadal, J.P., Isaac, P., Siñeriz, F., Ferrero, M.A., 2010. Indigo production by Pseudomonas sp. J26, a marine naphthalene-degrading strain. J. Basic Microbiol. 50, 290-293. https://doi.org/10.1002/jobm.200900276.

Ron, E.Z., Rosenberg, E., 2002. Biosurfactants and oil bioremediation. Curr. Opin. Biotechnol. 13, 249-252. https://doi.org/10.1016/S0958-1669(02)00316-6.

Ron, E.Z., Rosenberg, E., 2014. Enhanced bioremediation of oil spills in the sea. Curr. Opin. Biotechnol. 27, 191-194. https://doi.org/10.1016/j.copbio.2014.02.004.

Rosenberg, M., Gutnick, D., Rosenberg, E., 1980. Adherence of bacteria to hydrocarbons: a simple method for measuring cell-surface hydrophobicity. FEMS Microbiol. Lett. 9, 29-33. https://doi.org/10.1111/j.1574-6968.1980.tb05599.x.

Safdari, M.S., Kariminia, H.R., Rahmati, M., Fazlollahi, F., Polasko, A., Mahendra, S., Wilding, W.V., Fletcher, T.H., 2018. Development of bioreactors for comparative study of natural attenuation, biostimulation, and bioaugmentation of petroleum-hydrocarbon contaminated soil. J. Hazard Mater. 342, 270-278. https://doi.org/10. 1016/j.jhazmat.2017.08.044.

Schmutzler, K., Kracht, O.N., Schmid, A., Buehler, K., 2016. Trophic regulation of autoaggregation in Pseudomonas taiwanensis VLB120. Appl. Microbiol. Biotechnol. 100, 347-360. https://doi.org/10.1007/s00253-015-7006-2.

Smułek, W., Zdarta, A., Guzik, U., Dudzińska-Bajorek, B., Kaczorek, E., 2015. Rahnella sp. strain EK12: cell surface properties and diesel oil biodegradation after long-term contact with natural surfactants and diesel oil. Microbiol. Res. 176, 38-47. https:// doi.org/10.1016/j.micres.2015.04.008.

Teng, Y., Luo, Y., Ping, L., Zou, D., Li, Z., Christie, P., 2010. Effects of soil amendment with different carbon sources and other factors on the bioremediation of an aged PAH-contaminated soil. Biodegradation 21, 167-178. https://doi.org/10.1007/ s10532-009-9291-x.

Tian, W., Yao, J., Liu, R., Zhu, M., Wang, F., Wu, X., Liu, H., 2016. Effect of natural and synthetic surfactants on crude oil biodegradation by indigenous strains. Ecotoxicol. Environ. Saf. 129, 171-179. https://doi.org/10.1016/j.ecoenv.2016.03.027.

Velázquez-Torres, D., Chou-Rodríguez, E., Cabello-Eras, J.J., 2015. Chemical characterization of the corn steep water of UEB glucose cienfuegos and the one obtained using green sulf. Rev. Cubana Quím. 27, 275-288.

Yang, X., Chen, Z., Wu, Q., Xu, M., 2018. Enhanced phenanthrene degradation in river sediments using a combination of biochar and nitrate. Sci. Total Environ. 619-620, 600-605. https://doi.org/10.1016/j.scitotenv.2017.11.130.

Zhao, Q., Liu, Y., 2016. State of the art of biological processes for coal gasification wastewater treatment. Biotechnol. Adv. 34, 1064-1072. https://doi.org/10.1016/j. biotechadv.2016.06.005.

Zhao, Z., Selvam, A., Woon-Chung Wong, J., 2011. Effects of rhamnolipids on cell surface hydrophobicity of PAH degrading bacteria and the biodegradation of phenanthrene. Bioresour. Technol. 102, 3999-4007. https://doi.org/10.1016/j.biortech.2010.11. 088. 